\title{
Benefícios do aleitamento materno para a mulher e o recém nascido
}

\section{Benefits of breastfeeding for women and newborms}

Beneficios de la lactancia materna para mujeres y recién nacidos

Francisco Lucas Leandro de Sousa ORCID: https://orcid.org/0000-0003-2802-2378 Centro Universitário Maurício de Nassau, Brasil E-mail: lucasleandro2912@gmail.com

Rayssa Stéfani Sousa Alves

ORCID: https://orcid.org/0000-0002-9666-675X Pontifícia Universidade Católica de Goiás, Brasil E-mail: rayssastefani02@gmail.com

Airton César Leite

ORCID: https://orcid.org/0000-0001-7184-8488

Centro Universitário Santo Agostinho, Brasil E-mail: ainton.cesar2014@gmail.com

Mariana Pereira Barbosa Silva ORCID: https://orcid.org/0000-0003-0852-8099 Universidade Estadual do Piauí, Brasil E-mail: marianapbsilvaa@gmail.com

Celina Araújo Veras

ORCID: https://orcid.org/0000-0002-6542-0888 Centro Universitário UNINOVAFAPI, Brasil E-mail: celinaveras22@outlook.com

Ronnyele Cassia Araujo Santos ORCID: https://orcid.org/0000-0001-9203-2680

Centro Universitário Mauricio de Nassau, Brasil

E-mail: ronnyeleenfermagem@gmail.com

Rafaela Guimarães Freitas

ORCID: https://orcid.org/0000-0002-1112-3917

Universidade Do Estado Da Bahia, Brasil

E-mail: rafaellafreitas_02@hotmail.com

Vanessa Cristina Regis da Silva

ORCID: https://orcid.org/0000-0001-8415-5779

Universidade Federal do Triângulo Mineiro, Brasil E-mail: vanessacrisfisio@hotmail.com

Angelica Taciana Sisconetto

ORCID: https://orcid.org/0000-0001-6396-7372

Universidade Federal do Triângulo Mineiro, Brasil

E-mail: angelicasisconeto@ hotmail.com

Kelly Savana Minaré Baldo Sucupira

ORCID: https://orcid.org/0000-0003-1932-9458

Universidade Federal do Triângulo Mineiro, Brasil E-mail: kellyminare@gmail.com

Laíssa Almeida Custódio da Silva ORCID: https://orcid.org/0000-0003-3115-9375 Centro Universitário Fametro, Brasil E-mail: laissa020@gmail.com

Sidney Freires dos Santos ORCID: https://orcid.org/0000-0002-4112-3336 Centro Universitário Maurício de Nassau, Brasil

E-mail: sidneyfreire12@gmail.com

Samilly Lorâna Farias de Sousa ORCID: https://orcid.org/0000-0003-2647-9382 Universidade Potiguar, Brasil E-mail: Samilly.lorrana@yahoo.com.br

Mariana Albuquerque de Miranda Galdino ORCID: https://orcid.org/0000-0002-2170-8695 Universidade Estadual da Paraíba, Brasil

E-mail: mariechicoenfermagem@gmail.com

Maria dos Santos Fernandes ORCID: https://orcid.org/0000-0002-8746-6481 Centro Universitário Doutor Leão Sampaio, Brasil

E-mail: mariafernandes9378@gmail.com 


\author{
Daniela Marta da Silva \\ ORCID: https://orcid.org/0000-0001-5256-3698 \\ Universidade Federal do Triângulo Mineiro, Brasil \\ E-mail: daniellasilva_06@yahoo.com.br \\ Jéssika Roberta Firme de Moura Santos \\ ORCID: https://orcid.org/0000-0003-1268-2400 \\ Universidade Federal do Piauí, Brasil \\ E-mail: jessikafmsantos@gmail.com \\ Vitória Pires Alencar \\ ORCID: https://orcid.org/0000-0002-5548-9670 \\ Centro Universitário UNINOVAFAPI, Brasi \\ E-mail: vitoriapalencar@outlook.com \\ Barbara Rodrigues Ferreira \\ ORCID: https://orcid.org/0000-0003-1154-4364 \\ Universidade Federal do Pará, Brasil \\ E-mail: barbara.ferreira@altamira.ufpa.br
}

\title{
Resumo
}

Este estudo tem como objetivo realizar uma revisão bibliográfica acerca dos benefícios do aleitamento materno para a mulher e para o recém nascido. Trata-se de uma revisão integrativa de literatura, com abordagem qualitativa, realizada por meio da buscativa de artigos indexados na Biblioteca Virtual em Saúde (BVS), com o auxílio das seguintes bases de dados: Scientific Electronic Library Online (SCIELO), National Center for Biotechnology Information (PUBMED), Literatura Latino-Americana e do Caribe em Ciências da Saúde (LILACS). Os critérios de inclusão compreendem pesquisas de revisão da literatura disponíveis nos bancos de dados descritos, compreendidos entre os anos de 2015 a 2021. Como critérios de exclusão, não foram considerados artigos mediante a recompensação monetária, incompletos e não convergentes com este estudo. Após a aplicação dos critérios de inclusão e exclusão, restaram 12 artigos cientificos. Os resultados constata que, a prática do aleitamento materno é de fundamental importância para a mãe, a criança e a sociedade, devendo ser sempre incentivada e protegida. Constitui-se em uma sábia estratégia natural de vínculo, afeto, proteção e nutrição para a criança, gerando um grandioso impacto na promoção da saúde integral da dupla mãe/bebê e, consequentemente, na redução da morbimortalidade infantil e materna. Conclui-se que o leite materno é o alimento adequado para a criança tanto do ponto de vista nutritivo e imunológico quanto no plano psicológico, pois além de favorecer o vínculo mãe-filho, oferece os nutrientes que a criança necessita para iniciar uma vida saudável.

Palavras-chave: Aleitamento materno; Saúde da criança; Saúde da mulher; Promoção da saúde.

\begin{abstract}
This study aims to carry out a bibliographic review on the benefits of breastfeeding for women and newborns. It is an integrative literature review, with a qualitative approach, carried out by searching for articles indexed in the Virtual Health Library (VHL), with the help of the following databases: Scientific Electronic Library Online (SCIELO), National Center for Biotechnology Information (PUBMED), Latin American and Caribbean Literature in Health Sciences (LILACS). The inclusion criteria include literature review surveys available in the databases described, between the years 2015 to 2021 . As exclusion criteria, they were not considered articles for monetary reward, incomplete and not converging with this study. After applying the inclusion and exclusion criteria, 12 scientific articles remained. The results show that the practice of breastfeeding is of fundamental importance for the mother, child and society, and should always be encouraged and protected. It constitutes a wise natural strategy of bonding, affection, protection and nutrition for the child, generating a great impact in promoting the integral health of the mother / baby couple and, consequently, in reducing infant and maternal morbidity and mortality. It is concluded that breast milk is the appropriate food for the child, both from a nutritional and immunological point of view, as well as at a psychological level, since in addition to favoring the mother-child bond, it offers the nutrients that the child needs to start a healthy life.
\end{abstract}

Keywords: Breastfeeding; Child health; Women's health; Health promotion.

\section{Resumen}

Este estudio tiene como objetivo realizar una revisión bibliográfica sobre los beneficios de la lactancia materna para mujeres y recién nacidos. Se trata de una revisión integradora de la literatura, con enfoque cualitativo, realizada mediante la búsqueda de artículos indexados en la Biblioteca Virtual en Salud (BVS), con la ayuda de las siguientes bases de datos: Scientific Electronic Library Online (SCIELO), Centro Nacional de Información Biotecnológica ( PUBMED), Literatura Latinoamericana y del Caribe en Ciencias de la Salud (LILACS). Los criterios de inclusión incluyen encuestas de revisión de la literatura disponibles en las bases de datos descritas, entre los años 2015 a 2021. Como criterios de exclusión, no se consideraron artículos de recompensa monetaria, incompletos y no convergentes con este estudio. Tras aplicar los criterios de inclusión y exclusión, quedaron 12 artículos científicos. Los resultados muestran que la práctica de la lactancia materna es de fundamental importancia para la madre, el niño y la sociedad, y siempre debe ser fomentada y protegida. Constituye una sabia estrategia natural de vinculación, afecto, protección y nutrición para el niño, generando un gran impacto en la promoción de la salud integral de la pareja madre / bebé y, en consecuencia, en la reducción de la morbimortalidad infantil 
y materna. Se concluye que la leche materna es el alimento adecuado para el niño, tanto desde el punto de vista nutricional e inmunológico, como desde el punto de vista psicológico, ya que además de favorecer el vínculo madre-hijo, ofrece los nutrientes que el niño necesita comenzar una vida saludable.

Palabras clave: Amamantamiento; Salud de los niños; La salud de la mujer; Promoción de la salud.

\section{Introdução}

De acordo com a Organização Mundial da Saúde (OMS), aleitamento materno é o processo pelo qual o lactente recebe leite materno independentemente de consumir outros alimentos. $\mathrm{O}$ aleitamento materno exclusivo é o processo em que o bebê recebe leite materno de sua mãe ou nutriz ou leite materno extraído, sem receber nenhum outro líquido ou sólido, exceto vitaminas, complementos minerais ou medicamento (Furtado \& Assis, 2018).

A amamentação é uma prática antiga, reconhecida por seu benefício nutricional, imunológico, cognitivo, econômico e social. Tais benefícios são aproveitados em sua plenitude quando a amamentação é praticada por pelo menos dois anos e de forma exclusiva, até o sexto mês de vida (Furtado \& Assis, 2018).

A oferta do seio materno ao bebê é um direito biológico e eticamente inquestionável da mãe e do filho, além de fundamental para a sobrevivência e a qualidade de vida infantil nos primeiros anos de vida. Hoje, sabe-se que os benefícios do aleitamento materno não se limitam à duração da prática, mas se estendem até a vida adulta e têm repercussões na qualidade de vida a longo prazo (Ciampo \& Ciampo, 2018).

Nos últimos 30 anos, o Brasil tem promovido ações de promoção, proteção e apoio ao aleitamento materno, tendo em vista aumentar os índices de aleitamento exclusivo e complementar no país e inibir o desmame precoce (Azevedo et al., 2015). Em 1981, foi instituído o Programa Nacional de Incentivo ao Aleitamento Materno (PNIAM), na tentativa de intervir na mortalidade infantil (Furtado \& Assis, 2018).

A Política Nacional de Aleitamento Materno (PNAM) é organizada de acordo com as seguintes estratégias: Incentivo ao Aleitamento Materno na Atenção Básica - Rede Amamenta Brasil; Iniciativa Hospital Amigo da Criança (IHAC) e Método Canguru na atenção hospitalar; Rede Brasileira de Bancos de Leite Humano; Proteção legal através da Norma Brasileira de Comercialização de Alimentos para Lactentes (NBCAL); Ações de Mobilização Social através de campanhas e parcerias; Monitoramento das ações e práticas de aleitamento materno e, nos últimos anos, implantação da Iniciativa Unidade Básica Amiga da Amamentação (IUBAAM) (Azevedo et al., 2015).

Amamentar é muito mais do que nutrir a criança. É um processo que envolve interação profunda entre mãe e filho, com repercussões no estado nutricional da criança, em sua habilidade de se defender de infecções, em sua fisiologia e no seu desenvolvimento cognitivo e emocional, e em sua saúde no longo prazo, além de ter implicações na saúde física e psíquica da mãe (Brasil, 2015).

O leite materno é o alimento com maior quantidade de nutrientes e agentes imunológicos que protegem o recém nascido de infecções, sendo estas as principais causas de mortalidade neonatal (Campos, Gouveia, Strada \& Moraes, 2020).

Desse modo, o aleitamento materno imediato ao nascimento é essencial para o recém nascido, e evita que $22 \%$ dos neonatos morram por infecções, além de auxiliar na prevenção de hemorragias nas puérperas, que é a principal causa de morte materna atualmente. $\mathrm{O}$ aleitamento materno ainda traz benefícios de longo prazo para a criança, como maior rendimento escolar, maior quociente de inteligência e maior tempo de estudo (Campos, Gouveia, Strada \& Moraes, 2020).

É extremamente importante que os profissionais reconheçam a mulher como protagonista do seu processo de amamentar, valorizando-a, escutando-a e empoderando-a (Lima et al., 2019).

Sendo assim, quais são os benefícios do aleitamento materno? A importância desse estudo consiste em aprimorar o conhecimento acerca da temática proposta e identificar quais são as estratégias assistenciais do enfermeiro frente as orientações em saúde quanto ao benefício do aleitamento materno para a mulher e para o recém nascido. 
Este estudo tem como objetivo realizar uma revisão bibliográfica acerca dos benefícios do aleitamento materno para a mulher e para o recém nascido.

\section{Metodologia}

Trata-se de um estudo descritivo, do tipo revisão integrativa de literatura, de caráter qualitativo uma vez que é definida como um tipo de investigação voltada para o aspecto qualitativo de uma determinada questão, nesse caso, a importância e benefícios do aleitamento materno. A revisão de literatura permite aprofundar dentro de diversos autores e referenciais, sobre os discursos e principais temas abordados (Pereira et al., 2018).

A análise dos dados foi realizado entre setembro de 2020 a janeiro de 2021, mediante a revisão sistemática de artigos indexados e publicados na Plataforma da Biblioteca Virtual da Saúde (BVS), que compreende as bases de dados SciELO (Scientific Eletronic Library Online), LILACS (Literatura Latino-Americana e do Caribe em Ciências da Saúde), MEDLINE (Literatura Internacional em Ciências da Saúde), BDENF (Base de Dados de Enfermagem).

Foram usados os seguintes descritores em base DeCS (Descritores em Ciências da Saúde): Aleitamento materno; Saúde da Criança; Saúde da Mulher; Promoção da Saúde; Combinados com o operador booleando “AND” entre os descritores citados.

Os critérios de inclusão para a seleção dos conteúdos foram, artigos completos na linguagem portuguesa, inglesa e espanhola. Publicados na íntegra de acordo com a temática proposta, e artigos científicos publicados nos referidos bancos de dados compreendendo os anos de 2015 a 2021. Os critérios de exclusão foram, artigos que não tivessem relevância com a temática, materiais duplicados, incompletos, resumos, resenhas, debates, relato de caso, relato de experiência, publicados em anais de eventos e materiais indisponíveis na íntegra.

\section{Resultados e Discussão}

A partir da revisão de literatura e análise dos estudos indexados nas bases de dados eletrônicas, acerca da temática proposta, foram encontrados 212 estudos científicos, sendo que, apenas 28 estudos foram selecionados, 22 atenderam aos critérios de inclusão previamente estabelecidos, destes, 10 foram excluídos com base nos critérios de exclusão, restando 12 artigos para composição e análise do estudo. O fluxograma com o detalhamento das etapas de pesquisa está apresentado a seguir na Figura 1. 
Figura 1. Fluxograma de identificação e seleção dos artigos. 2020.
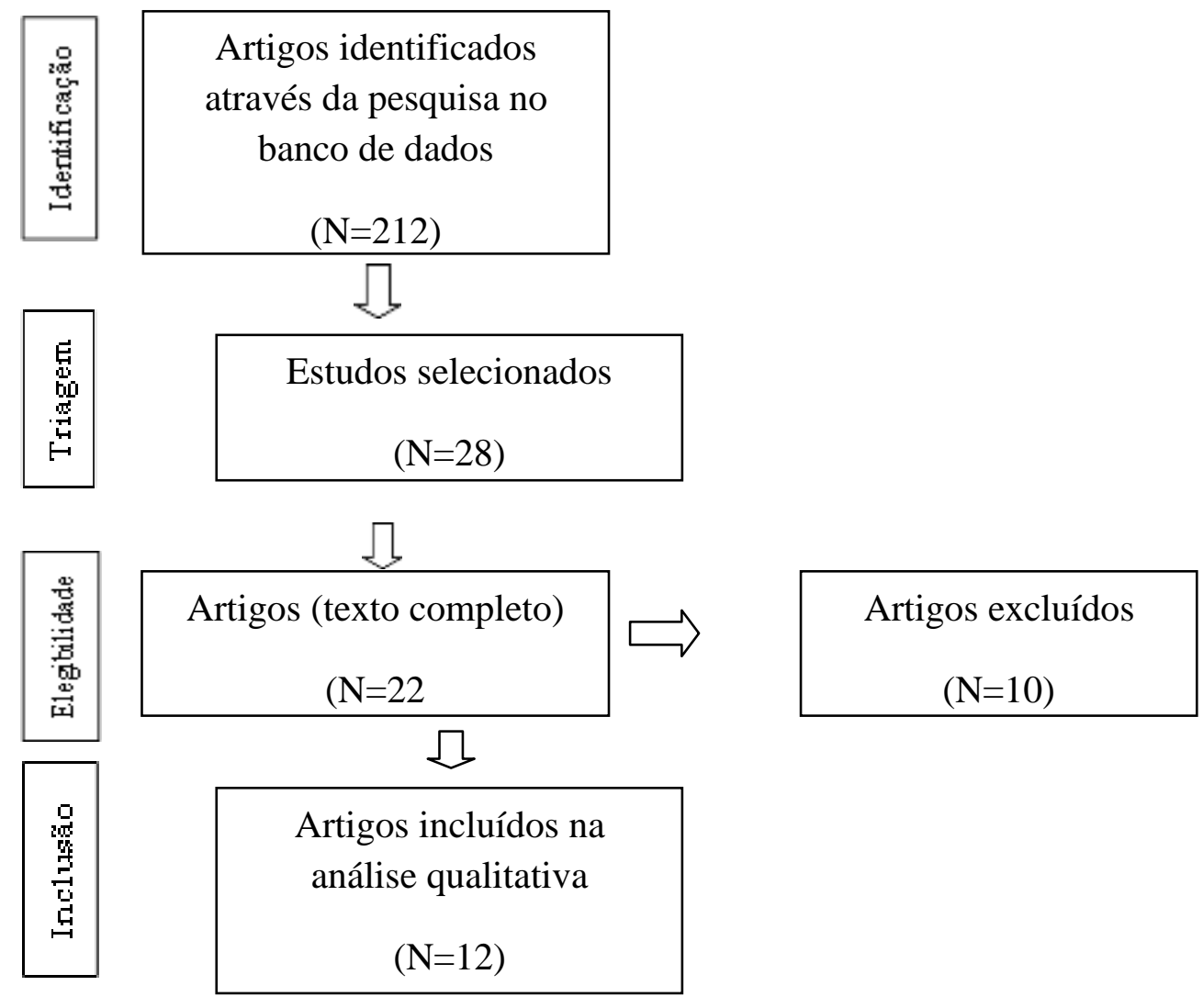

Fonte: Elaboração Própria (2021).

O leite materno é o alimento mais adequado para promover o desenvolvimento e crescimento eficaz da criança. A Organização Mundial da Saúde (OMS) juntamente com o Ministério da Saúde recomenda que ele deve ser ofertado de forma exclusiva nos seis primeiros meses de vida da criança e sob livre demanda. Sendo que após esse período o aleitamento materno poderá ser complementado com outros tipos de alimentos até os dois anos de idade ou mais (Brasil, 2015).

A lactação é uma característica diferencial dos mamíferos. A síntese e a secreção do leite são processos bioquímicos e neuroendócrinos complexos sob controle hormonal e envolvem os terminais sensíveis da aréola e do mamilo. A lactação é o resultado direto e natural da gravidez e do nascimento e parte integrante do processo reprodutivo que beneficia mãe e filho simultaneamente. A interação desses fatores culmina com a produção de leite, causa mudanças no organismo materno e favorece boas condições de saúde física e emocional para a mãe lactante, que se estenderão para a sua vida futura (Ciampo \& Ciampo, 2018).

O leite humano contém água em quantidade suficiente; proteína e gordura mais adequadas para a criança, além de vitaminas em quantidades suficientes, dispensando o uso de suplementos vitamínicos, assim protegendo contra alergias e infecções, especialmente as diarreias, favorecendo o crescimento e desenvolvimento da criança (Azevedo et al., 2015).

A prática do aleitamento materno é de fundamental importância para a mãe, a criança e a sociedade, devendo ser sempre incentivada e protegida. Constitui-se em uma sábia estratégia natural de vínculo, afeto, proteção e nutrição para a criança, gerando um grandioso impacto na promoção da saúde integral da dupla mãe/bebê e, consequentemente, na redução da morbimortalidade infantil e materna (Lima et al., 2019). 
É através da amamentação que a criança vivencia estímulos diversos e a interação estabelecida a cada mamada propicialhe a consolidação de sentimentos de segurança, proteção e bem estar, os quais são fundamentais para um desenvolvimento infantil saudável. A proximidade entre os corpos permite a criança perceber os batimentos cardíacos, a temperatura e a respiração materna (Macedo et al., 2015).

Estabelece-se um bem estar físico, onde o lactente se sente aconchegado no seio materno. Além da sensação de proteção, o contato com a pele, exerce também um impacto positivo no desenvolvimento emocional da criança, tornando-a mais calma e tranquila. Forma-se, um verdadeiro elo de afetividade, o qual é imprescindível para o desenvolvimento mental e psíquico do lactente (Macedo et al., 2015).

Os benefícios do contato pele a pele (CPP) para o recém nascido são melhor efetividade da primeira mamada, redução do tempo de desenvolver uma sucção eficaz, regulação e manutenção da temperatura corporal do recém nascido e estabilidade cardiorrespiratória (Campos, Gouveia, Strada \& Moraes, 2020).

Já para a mulher, ocorre diminuição da dor causada pelo ingurgitamento mamário, sentimento de alívio, segurança e diminuição da ansiedade desenvolvida ao longo da gestação (Campos, Gouveia, Strada \& Moraes, 2020).

Além disso, o contato pele a pele pode resultar em melhores índices de aleitamento materno nos primeiros quatro meses após o parto, maior duração do aleitamento materno, melhor comportamento de afeto e apego da mãe, vínculo, sentimentos de felicidade, amor, tranquilidade e conforto para a mulher e o recém nascido. Essa mistura de sentimentos faz com que a mulher desvie sua atenção do desconforto e da dor do parto para o prazer de estar com o seu recém nascido (Campos, Gouveia, Strada \& Moraes, 2020).

O aleitamento materno exclusivo nos primeiros 6 meses de vida do bebê é de suma importância, pois garante nutrição adequada, ajuda no desenvolvimento das estruturas orais, como lábios, língua, bochechas, palato duro e mole, responsáveis pelo funcionamento adequado das funções de respiração, sucção, mastigação, deglutição e fonoarticulação, além de propiciar o padrão de respiração nasal (Rosa \& Delgado, 2017).

Além de todas as vantagens para as crianças, o aleitamento materno traz também importantes benefícios para a saúde da mulher (Peres, Cascaes, Nascimento \& Victora, 2015).

Mulheres que amamentam recuperam mais rapidamente o peso que possuíam antes da gravidez, além de possuírem menor risco de hemorragias no puerpério imediato e consequentemente anemia por perda sanguínea, ressalta-se ainda, uma maior proteção contra o desenvolvimento de câncer de mama, entre outros (Chowdhury et al., 2015).

$\mathrm{O}$ aleitamento materno também inclui vantagens de curto e longo prazo para o recém nascido e para as mulheres. O aleitamento materno imediatamente após o nascimento, em curto prazo, previne morbidade e mortalidade neonatal, estando associado ao maior tempo de duração do aleitamento materno e maior tempo de aleitamento materno exclusivo (Campos, Gouveia, Strada \& Moraes, 2020).

Para a mulher, favorece a liberação de ocitocina e tem efeito protetor nos transtornos do estado de ânimo materno. Em longo prazo, entre os benefícios, está o melhor desenvolvimento motor dos recém nascidos, além da diminuição do risco de doenças, como: diabetes, obesidade, gastroenterite, entre outras. A mulher apresenta amenorréia lactacional, diminuição de risco de desenvolver diabetes tipo 2, cânceres de ovário e de mama, além de perder peso mais rápido (Campos, Gouveia, Strada \& Moraes, 2020).

Segundo evidências científicas a prática de amamentar o bebê exclusivamente com leite materno até o sexto mês de vida promove a prevenção de várias doenças tais como: diarreia e outras doenças intestinais, infecções respiratórias, infecções bacterianas, infecções do trato urinário, alergias, infecções hospitalares, melhor padrão cardiorrespiratório durante a alimentação, melhor resposta às imunizações e proteção contra as Doenças Crônicas Não Transmissíveis (DCNTs) (Taveiro, Vianna \& Pandolfi, 2020). 
O aleitamento materno favorece o crescimento e o desenvolvimento da criança, tanto por suas características nutricionais, imunológicas e psicológicas, quanto por possibilitar o crescimento harmonioso da face, promovendo a maturação das funções do sistema estomatognático. A amamentação é recomendada de forma exclusiva até o sexto mês de vida e de forma complementada até dois anos ou mais, tornando desnecessário o uso de mamadeiras com qualquer tipo de líquido (Teles, Junior, Júnior, Fonseca \& Eugênio, 2017).

Azevedo et al. (2015) aborda que amamentar não é um processo simples e que envolve questões sociais, biológicas, psicológicas e culturais. Deve-se respeitar os desejos e decisões maternas, orientando-as visando garantir a melhor alimentação para o recém-nascido. Sendo assim, o manejo clínico da amamentação deve ser iniciado ainda no pré-natal, período em que a mulher já vai compreendendo a fisiologia da lactação, os benefícios para si e para o bebê durante a amamentação, dos intervalos entre as mamadas, dos sinais de hipoglicemia, o que lhe permite chegar à maternidade com esses conhecimentos. Se a orientação correta começar precocemente, as intervenções tenderão a diminuir quando a amamentação tiver sido iniciada.

Uma das características mais relevantes no pré-natal são o vínculo e o acolhimento das gestantes junto aos profissionais de saúde, devido ao corpo de conhecimento que o profissional dispõe com a associação de um conhecimento clínico, evidências científicas, para identificar e abordar particularidades de cada mulher. O vínculo estabelecido e fortalecido permite compreender necessidades, capacidades e limitações da mulher na compreensão do processo gestacional e do nascimento (Alves, Couto, Barreto \& Quitete, 2020)

Cabe aos profissionais da saúde a tarefa de garantir, a cada mãe, uma escuta ativa, ou seja, saber ouvi-la, diminuir suas dúvidas, entendê-las e esclarecê-las sobre suas crenças e tabus, de modo a tornar a amamentação um ato de prazer e não o contrário. É importante que as mães se sintam encorajadas a prosseguir com o aleitamento natural (Furtado \& Assis, 2018).

\section{Considerações Finais}

Este estudo confirmou a importância do aleitamento materno para a mulher e o recém nascido, considerando que o leite materno é o alimento adequado para a criança tanto do ponto de vista nutritivo e imunológico quanto no plano psicológico, pois além de favorecer o vínculo mãe-filho, oferece os nutrientes que a criança necessita para iniciar uma vida saudável, sendo essencial para o lactente até o sexto mês de vida como alimento único e exclusivo, a partir de então, complementado com outras fontes nutricionais até pelo menos dois anos de idade. É importante que os profissionais da saúde incentivem e orientem as mães quanto aos beneficios do aleitamento materno, fornecendo as informações necessárias para que a prática do aleitamento seja fortalecida.

Este estudo não contribui somente para a formação acadêmica, mas sugere e estimula a produção de novas pesquisas de temática similar que abordem a importância do aleitamento materno para que se possa contribuir futuramente na melhor qualidade de vida das pessoas.

\section{Referências}

Alves, Y. R., Couto, L. L., Barreto, A. C. M., \& Quitete, J. B. (2020). A amamentação sob a égide de redes de apoio: uma estratégia facilitadora. Escola Anna Nery, 24(1), e20190017.

Azevedo, A. R. R., Alves, V. H., Souza, R. M. P., Rodrigues, D. P., Branco, M. B. L. R., \& Cruz, A. F. N. (2015). O manejo clínico da amamentação: saberes dos enfermeiros. Escola Anna Nery, 19(3), 439-445.

Campos, P. M., Gouveia, H. G., Strada, J. K. R., \& Moraes, B. A. (2020). Contato pele a pele e aleitamento materno de recém-nascidos em um hospital universitário. Revista Gaúcha de Enfermagem, 41(spe), e20190154.

Chowdhury, R., Sinha, B., Sankar, M. J., Taneja, S., Bhandari, N., Rollins, N., Bahl, R., \& Martines, J. (2015). Breastfeeding and maternal health outcomes: a systematic review and meta-analysis. Acta paediatrica, 104(467), 96-113.

Ciampo, L. A. D., \& Ciampo, I. R. L. D. (2018). Breastfeeding and the Benefits of Lactation for Women's Health. Revista Brasileira de Ginecologia e Obstetrícia, 40(6), 354-359

Furtado, L. C. R., \& Assis, T. R. (2018). Diferentes fatores que influenciam na decisão e na duração do aleitamento materno: Uma revisão da literatura. Movimenta, 
Research, Society and Development, v. 10, n. 2, e12710211208, 2021

(CC BY 4.0) | ISSN 2525-3409 | DOI: http://dx.doi.org/10.33448/rsd-v10i2.11208

5(4), 303-312.

Lima, S., Santos, E., Erdmann, A., Farias, P., Aires, J., \& Nascimento, V. (2019). Percepção de mulheres quanto à prática do aleitamento materno: uma revisão integrativa. Revista de Pesquisa: Cuidado é Fundamental Online, 11(1), 248-254.

Macedo, M. D. S., Torquato, I. M. B., Trigueiro, J. V. S., Albuquerque, A. M., Pinto, M. B., \& Nogueira, M. F. (2015). Aleitamento materno: identificando a prática, benefícios e os fatores de risco para o desmame precoce. Journal of Nursing UFPE on line, 9(1), 414-423.

Ministério da Saúde. (2015). Saúde da criança: aleitamento materno e alimentação complementar. (2a ed.) Cadernos de Atenção Básica. Secretaria de Atenção à Saúde. Departamento de Atenção Básica. Brasília, DF: Ministério da Saúde.

Pereira, A. S., et al. (2018). Metodologia da pesquisa científica. UAB/NTE/UFSM. https://repositorio.ufsm.br/bitstream/handle/1/15824/Lic_ Computacao_Metodologia-Pesquisa-Cientifica.pdf?sequence=1.

Peres, K. G., Cascaes, A. M., Nascimento, G. G., \& Victora, C. G. (2015). Effect of breastfeeding on malocclusions: a systematic review and meta-analysis. Acta paediatrica (Oslo, Norway: 1992), 104(467), 54-61.

Rosa, J. B. S., \& Delgado, S. E. (2017). Conhecimento de puérperas sobre amamentação e introdução alimentar. Rev Bras Promoç Saúde, $30(4), 1-9$.

Taveiro, E. A. N., Vianna, E. Y. S., \& Pandolfi, M. M. (2020). Adesão ao Aleitamento Materno Exclusivo em Bebês de 0 a 6 Meses Nascidos em um Hospital e Maternidade do Município de São Paulo. Rev Bras Ciên Saúd, 24(1), 71-82.

Teles, M. A. B., Junior, R. F. S., Júnior, G. G. S., Fonseca, M. P., \& Eugênio, K.K. (2017). Conhecimento e práticas de aleitamento materno de usuárias da estratégia saúde da família. Rev enferm UFPE on line., 11(6), 2302-2308. 